\title{
PREVALÊNCIA DOS DISTÚRBIOS OSTEOMUSCULARES RELACIONADOS AO TRABALHO: estudo com lavadeiras de roupa
}

\author{
PREVALENCE OF CUMULATIVE TRAUMA DISORDERS: \\ study with clothes washerwomen
}

\section{Elenir Alcântara Diniz' , Rafaella do Carmo Ribeiro², Milena Nunes Alves de Sousa ${ }^{3}$}

\begin{abstract}
RESUMO
Objetivou-se avaliar a prevalência de distúrbios osteomusculares relacionados ao trabalho em lavadeiras no município de Caicó-RN. Estudo de campo, exploratório-descritivo com abordagem quantitativa foi realizado com uma amostra de 45 lavadeiras, determinada mediante amostra não probabilística por conveniência. Os dados foram coletados a partir de questionários e analisados no SPSS 21.0. Utilizou-se de estatísticas descritivas de frequência e porcentagem, além de média, desvio padrão, mediana, valores mínimos e máximos. Adicionalmente, foram utilizados testes inferenciais. Observou-se a exclusividade de mulheres trabalhadoras e constatou-se a maior frequência de acometimento por distúrbios osteomusculares nas regiões de mãos/punho e cotovelos (22,2\% e 46,7\%). Quanto aos problemas osteomusculares relacionados ao trabalho, o mais associado foi o dos punhos ou mãos $(95,6 \%)$, seguido dos cotovelos $(62,2 \%)$ e pescoço/região cervical $(53,3 \%)$. Houve queixas osteomusculares em toda a amostra, havendo correlação entre as regiões acometidas e seu uso para a realização das atividades laborais desenvolvidas pelas lavadeiras.
\end{abstract}

Descritores: Saúde do Trabalhador; Riscos Ocupacionais; Transtornos Traumáticos Cumulativos.

\section{ABSTRACT}

This study aimed to assess the prevalence of musculoskeletal disorders related to work washerwomen in the city of Caico-RN. Field of study, exploratory and descriptive with quantitative approach was conducted with a sample of 45 washerwomen, determined by non-probabilistic convenience sample. Data were collected from questionnaires and analyzed by SPSS. We used descriptive statistics of frequency and percentage, as well, the mean, standard deviation, median, minimum and maximum values. Additionally, inferential tests were used. There was the uniqueness of working women and found that the higher frequency of involvement by musculoskeletal disorders in the areas of hand / wrist and elbows ( $22.2 \%$ and 46.7\%). As for musculoskeletal problems related to work, the more associated was the wrists or hands $(95.6 \%)$, followed by elbows $(62.2 \%)$ and neck / cervical region $(53.3 \%)$. There were musculoskeletal complaints in the whole sample, there is correlation between the regions involved and its use for the realization of the developed industrial activities of washerwomen.

Descriptors: Occupational Health; Occupational Risks; Cumulative Trauma Disorders.
${ }^{1}$ Enfermeira pela Faculdade Integrada de Patos (FIP), Patos, $\mathrm{PB}$, Brasil.

${ }^{2}$ Graduanda em Medicina pela Faculdade Integrada de Patos (FIP), Patos, PB, Brasil.

${ }^{3}$ Pós-Doutora em Promoção de Saúde pela Universidade de Franca (UNIFRAN), Franca, SP, Brasil. 


\section{Introdução}

O trabalho é considerado uma atividade fundamental de emancipação do sujeito ao longo da história ${ }^{1}$. No entanto, denuncia o processo de exploração ao qual são submetidos os trabalhadores. Dessa forma, acredita-se que deixa de ser uma atividade emancipatória, pois é responsável por uma grande parcela das situações de adoecimento dos indivíduos trabalhadores².

Nesse contexto, o aumento da exposição a fatores de risco e a ampliação da vulnerabilidade dos trabalhadores acabam desencadeando agravos à saúde e à segurança, o que pode ocasionar cenários de adoecimento como consequência das más condições de trabalho ${ }^{3}$.

Entre os principais agravos que os trabalhadores são acometidos, estão os Distúrbios Osteomusculares Relacionados ao Trabalho (DORTs) que representam um grupo de afecções musculoesqueléticas relacionadas ao trabalho de origem multicausal, complexa e de caráter insidioso no que diz respeito ao aparecimento e evolução. Sua instalação combina fatores biomecânicos da atividade como tensão/fricção sobre os tendões; posturas inadequadas no posto de trabalho; exposição a temperaturas extremas e outros aspectos psicossociais da organização e dinâmica do trabalho, como monotonia da tarefa, expropriação do controle sobre a atividade, percepção individual sobre a carreira e relacionamento com a equipe de trabalho ${ }^{4}$.

De acordo com a norma técnica $n^{0}$. 606/1998 do Instituto Nacional do Seguro Social (INSS), as DORTs consistem em "[...] lesões por esforços repetitivos como uma síndrome clínica caracterizada por dor crônica, acompanhada ou não e alterações objetivas, que se manifesta principalmente no pescoço, cintura escapular e/ou membros superiores em decorrência do trabalho, podendo afetar tendões, músculos e nervos periféricos":715 .

O diagnóstico de DORTs é complexo e de difícil execução, pois engloba um conjunto de fatores e o nexo com o trabalho questionável, já que a instalação da patologia consiste em um processo social. Inicialmente, os casos do agravo no Brasil foram descritos como tenossinovites ocupacionais ${ }^{5}$. Este agravo também tem sido comum em lavadeiras. Já em 1973, no XII Congresso Nacional de Prevenção de Acidentes do Trabalho, foram apresentados dados sobre casos de tenossinovites ocupacionais em lavadeiras, limpadoras e engomadeiras, recomendando-se que fossem observadas pausas de trabalho para aqueles que operavam intensamente com as mãos ${ }^{5}$.

A atividade realizada pelas lavadeiras parece desencadear lesões osteomusculares decorrentes das condições de trabalho que exigem esforços dos seus membros superiores, pois é repetitiva. Além disso, há sobrecarga estática das estruturas desses membros e existe aparente tensão exigida para desenvolver as atividades. Há, ainda, movimentação anormal das mãos ou movimentos violentos e irregulares, assim como posturas inadequadas ao executar o trabalho, ocasionando uma incidência de sintomas, tais como: fortes dores nos braços, formigamentos, dificuldades de movimentos e, consequentemente, perda da força nas mãos e desenvolvimento de lesões ${ }^{6}$.

$O$ adoecimento desses profissionais leva a uma modificação negativa na sua qualidade de vida, uma vez que o seu afastamento do trabalho provoca impacto em suas condições sociais e financeiras. Dessa forma, vê-se a necessidade de estudos relacionados ao impacto dessas lesões na vida desse grupo, para assim, propor ações que minimizem a exposição aos riscos e agravos relacionados ao trabalho, especialmente as DORTs.

Sabe-se que no município de Caicó/RN há um grande número de lavadeiras e engomadeiras de roupa, contudo, não há trabalhos científicos ou dados disponíveis sobre a situação de trabalho e saúde das mesmas. Nessa ótica e considerando os riscos aos quais estão expostas diariamente, indaga-se: qual a prevalência de distúrbios osteomusculares relacionados ao trabalho em lavadeiras de roupa no município de Caicó-RN?

O presente trabalho teve como objetivo geral avaliar a prevalência de distúrbios osteomusculares relacionados ao trabalho em lavadeiras no município de Caicó-RN. De modo específico, buscou-se caracterizar o perfil social e demográfico das lavadeiras, identificar as lesões mais prevalentes e verificar as principais mudanças sociais e econômicas após 0 aparecimento da doença.

\section{Metodologia}

Estudo de campo, do tipo exploratório-descritivo com abordagem quantitativa, o cenário foi o município de Caicó, cidade localizada no interior do Estado do Rio Grande do Norte há $282 \mathrm{~km}$ da capital do Estado, apresentando uma população de aproximadamente 62.709 habitantes. 
Participaram da pesquisa 45 lavadeiras ( $90 \%$ do universo de pesquisa), que executam suas tarefas diariamente, com lavagem de pelo menos 2 a 3 "trouxas de roupa", aproximadamente $40 \mathrm{~kg}$, com uma jornada de trabalho que tem início às cinco horas da manhã e é finalizada às dezessete horas, com pausa de duas horas para almoço. Ressalta-se que lavadeiras envolvidas nesta atividade não executam outra atividade remunerada.

Portanto, a amostra adotada foi do tipo não probabilístico por conveniência, a qual foi estabelecida mediante adequação aos critérios de inclusão e exclusão. Incluíram-se as lavadeiras de roupa residentes no município de Caicó$\mathrm{RN}$, com 18 anos ou mais de idade e as quais aceitaram voluntariamente em participar da pesquisa assinando o Termo de Consentimento Livre e Esclarecido. Por conseguinte, excluíram-se aquelas profissionais que estavam doentes durante 0 período de coleta de dados, bem como aquelas que não estavam cadastradas na Prefeitura Municipal local, uma vez que só é possível realizar a lavagem de roupas na lavanderia do município aquelas profissionais cadastradas.

A coleta de dados foi realizada no período de junho a outubro de 2015 após parecer favorável do Comitê de Ética em Pesquisa das Faculdades Integradas de Patos - CAAE: 45395615.3.0000.5181/ Número do Parecer: 1.215.455/2015. Foram aplicados três instrumentos: 1) Questionário socioeconômico, com questões objetivas e subjetivas, elaboradas pela autora; 2) o Questionário Preditivo quanto aos Fatores de Risco para LER/DORTs, constando somente questões objetivas $^{7}$; e 3) Questionário Nórdico de Sintomas Osteomusculares (QNSO), abrangendo os aspectos organizacionais, psicossociais e ergonômicos ${ }^{8}$.

Os dados foram analisados no Statistical Package for the Social Sciences (SPSS), versão 21. Utilizou-se de estatísticas descritivas de frequência e porcentagem, além de média, desvio padrão, mediana, valores mínimos e máximos. Como teste inferencial utilizou-se a correlação de Pearson e aceitou-se como estatisticamente significativo um $p<0,05$.

\section{Resultados e Discussão}

A amostra foi composta em sua totalidade pelo sexo feminino (100\%). A tabela 1 mostra que a maioria da amostra tem apenas o ensino fundamental $(91,1 \%)$, é solteira $(44,4 \%)$ e não possui doenças $(61,4 \%)$. Entre aquelas que relataram possuir doença, a mais prevalente foi a hipertensão (17,8\% da amostra).

Ainda, é possível visualizar na tabela 1 que uma pequena maioria da amostra possuía entre 38 a 50 anos, com média de idade de 39,87 anos ( $D P=10,47)$, renda entre 608 a 788 reais, com média de 629,38 reais $(D P=162,73)$. Em relação ao tempo de trabalho, a maioria possuía entre 10 e 23,99 anos, com tempo médio de 19,76 anos (DP = 7,93).

Tabela 1 - Descrição dos dados sociais e demográficos. Caicó, RN, Brasil, 2015.

\begin{tabular}{c|c|c|c}
\hline \multirow{2}{*}{ Escolaridade } & Variáveis & F & $\%$ \\
\cline { 2 - 4 } & Mundamental & 41 & 91,1 \\
\hline \multirow{4}{*}{ Estado civil } & Solteiro & 4 & 8,9 \\
\cline { 2 - 4 } & Casado ou união estável & 16 & 44,4 \\
\cline { 2 - 4 } & Divorciado & 1 & 35,6 \\
\cline { 2 - 4 } & Viúvo (a) & 8 & 17,8 \\
\hline \multirow{4}{*}{ Idade } & Entre 25 e 37 anos & 18 & 40,0 \\
\cline { 2 - 4 } & Entre 38 a 50 anos & 22 & 48,9 \\
\cline { 2 - 4 } & Entre 51 a 64 anos & 5 & 11,1 \\
\hline \multirow{4}{*}{ Renda } & Entre 250 a 429 anos & 8 & 17,8 \\
\cline { 2 - 4 } & Entre 429 a 607 & 18 & 40,0 \\
\cline { 2 - 4 } & Entre 608 a 788 & 19 & 42,2 \\
\hline \multirow{4}{*}{ Tempo de trabalho } & Entre 10 e 23,99 anos & 35 & 77,8 \\
\cline { 2 - 4 } & Entre 24 a 37,99 anos & 9 & 20,0 \\
\cline { 2 - 4 } & Entre 38 a 52 anos & 1 & 2,2 \\
\hline
\end{tabular}




\begin{tabular}{c|c|c|c}
\hline \multicolumn{2}{|l|}{ Variáveis } & F & $\%$ \\
\hline \multirow{3}{*}{ Possui alguma doença } & Sim & 17 & 38,6 \\
\cline { 2 - 4 } & Não & 28 & 61,4 \\
\hline \multirow{4}{*}{ Qual } & Nenhuma & 28 & 61,4 \\
\cline { 2 - 4 } & Ansiedade & 3 & 6,7 \\
\cline { 2 - 4 } & Depressão & 3 & 6,7 \\
\cline { 2 - 4 } & Diabetes & 1 & 2,2 \\
\cline { 2 - 4 } & Hipertensão & 8 & 17,8 \\
\cline { 2 - 4 } & Hipertensão e diabetes & 1 & 2,2 \\
\cline { 2 - 4 } & Tireoide & 1 & 2,2 \\
\hline
\end{tabular}

Fonte: Dados de Pesquisa, 2015.

Apredominância de mulheres no serviço está relacionada a questões culturais e, por desenvolverem trabalho doméstico, além das atividades laborais, este público apresenta maior predisposição ao desgaste físico e mental ${ }^{9}$, especialmente em detrimento da jornada dupla de trabalho. "Idade e sexo são quesitos importantes no contexto laboral referente a LER/ DORT"10:13. Dentre os diversos campos da atividade humana, o trabalho das lavadeiras representa um tradicional reduto histórico feminino, já que o objeto principal é o trabalho doméstico, tradicionalmente exercido pelas mulheres.

No tocante à baixa escolaridade, acredita-se que pode estar relacionado com a necessidade vivenciada por muitas mulheres de, ainda, durante a juventude, precisarem abandonar os estudos para ingressar no mercado de trabalho e ajudarem nas despesas do lar ${ }^{11}$. Também, a baixa escolaridade de mulheres também pode estar relacionada ao matrimônio e maternidade precoces, por exemplo.

Em relação ao tempo de trabalho, autores ressaltam que estão susceptíveis ao acometimento pelas DORTs tanto indivíduos em início de carreira, bem como, aqueles que atuam há anos ${ }^{13}$.

Já os níveis de renda associam-se aos padrões de qualidade de vida, sendo esta considerada a interpretação do indivíduo em relação a sua posição na vida, no contexto da cultura e no sistema de valores em que vive, bem como aos seus objetivos, expectativas, padrões e preocupações ${ }^{13}$.

A tabela 2 faz uma descrição da frequência e das regiões com problemas osteomusculares. Verificou-se que a região do punho ou mãos/dedos foi a que apresentou maior frequência de "sempre" e "com frequência" sentiu problemas nessa região (respectivamente $22,2 \%$ e 46,7\%), seguido dos cotovelos (respectivamente $11,1 \%$ e 33,3\%).

Tabela 2 - Descrição da frequência e locais de problemas osteomusculares. Caicó, RN, Brasil, 2015.

\begin{tabular}{c|c|c|c|c}
\hline Regiões do Corpo & Não & Raramente & Com frequência & Sempre \\
\hline Pescoço/Região Cervical & $19(42,2 \%)$ & $18(40 \%)$ & $8(17,8 \%)$ & $0(0,0 \%)$ \\
\hline Ombros & $38(84,4 \%)$ & $2(4,4 \%)$ & $4(8,9 \%)$ & $1(2,2 \%)$ \\
\hline Braços & $31(68,9 \%)$ & $5(11,1 \%)$ & $8(17,8 \%)$ & $1(2,2 \%)$ \\
\hline Cotovelos & $16(35,6 \%)$ & $9(20 \%)$ & $15(33,3 \%)$ & $5(11,1 \%)$ \\
\hline Antebraços & $34(75,6 \%)$ & $1(2,2 \%)$ & $7(15,6 \%)$ & $396,7 \%)$ \\
\hline Punho ou Mãos ou dedos & $4(8,9 \%)$ & $10(22,2 \%)$ & $21(46,7 \%)$ & $10(22,2 \%)$ \\
\hline Região dorsal & $44(97,8 \%)$ & $0(0,0 \%)$ & $0(0,0 \%)$ & $1(2,2 \%)$ \\
\hline Região Lombar & $43(95,6 \%)$ & $1(2,2 \%)$ & $1(2,2 \%)$ & $0(0,0 \%)$ \\
\hline Quadril ou membros inferiores & $42(93,3 \%)$ & $2(4,4 \%)$ & $1(2,2 \%)$ & $0(0,0 \%)$ \\
\hline
\end{tabular}

Fonte: Dados de Pesquisa, 2015. 
"Problemas de saúde relacionados ao trabalho são comuns entre trabalhadores"13:131. Portanto, os achados parecem corroborar com o fato de existir a possibilidade de acometimento por problemas osteomusculares de todas as regiões do corpo, enfatizando, porém, sua ocorrência nos membros superiores ${ }^{15}$.

Resultados semelhantes ao deste trabalho foram também observados em outra pesquisa referente à prevalência de sintomas osteomusculares entre trabalhadores de um Serviço de Nutrição Hospitalar em São Paulo ${ }^{16}$. Neste estudo foi descrita a ocorrência de dor ou desconforto "sempre" nas regiões de mãos/punhos (29\%) e cotovelos (10\%).

Há presença de queixas osteomusculares em grande parte da população. Estudo com outra categoria profissional, a exemplo da enfermagem, indicou alta prevalência de DORT no grupo, com relatos de tais queixas em 96,3\% da amostra ${ }^{17}$. $\mathrm{O}$ achado reforça que tanto enfermeiros como lavadeiras utilizam em demasia o sistema osteomioarticular para execução das tarefas laborais diárias.

Também, outra pesquisa ${ }^{16}$ apontou presença de queixas em $89 \%$ da amostra de um grupo de nutricionistas. Ademais, estudo realizado com mototaxistas citou que a maior parte da amostra relatava queixas em mais de uma região corpórea ${ }^{11}$.

A tabela 3 apresenta a descrição da frequência e locais de problemas osteomusculares relacionados ao trabalho. O problema mais associado foi o dos punhos ou mãos (95,6\%), seguido do problema nos cotovelos $(62,2 \%)$ e no pescoço/região cervical (53,3\%).

Tabela 3 - Frequência e locais de problemas osteomusculares relacionados ao trabalho. Caicó, RN, Brasil, 2015.

\begin{tabular}{c|c|c|c|c}
\hline $\begin{array}{c}\text { Locais de problemas osteomusculares } \\
\text { relacionados ao trabalho }\end{array}$ & \multicolumn{2}{|c|}{ Não } & \multicolumn{2}{c}{ Sim } \\
\hline Nenhum problema & 45 & 100,0 & 0 & 0,0 \\
\hline Problemas no pescoço/região cervical & 21 & 46,7 & 24 & 53,3 \\
\hline Problemas nos ombros & 38 & 84,4 & 7 & 15,6 \\
\hline Problemas nos braços & 33 & 73,3 & 12 & 26,7 \\
\hline Problemas nos cotovelos & 17 & 37,8 & 28 & 62,2 \\
\hline Problemas nos antebraços & 35 & 77,8 & 10 & 22,2 \\
\hline Problemas nos punhos/mãos/dedos & 2 & 4,4 & 43 & 95,6 \\
\hline Problemas na região dorsal & 45 & 100,0 & 0 & 0,0 \\
\hline Problemas na região lombar & 43 & 95,6 & 2 & 4,4 \\
\hline Problemas no quadril/membros inferiores & 42 & 93,3 & 3 & 6,7 \\
\hline
\end{tabular}

Fonte: Dados de Pesquisa, 2015.

Nessa mesma perspectiva, a presença de DORTs engloba em sua caracterização as manifestações dolorosas que acometem, geralmente, as regiões do pescoço, cintura escapular e membros superiores ${ }^{4}$.

A região das mãos e punhos teve predominância nas queixas de acometimentos. Em estudo sobre a prevalência de sintomas osteomusculares em operadores de máquinas de colheita florestal foi identificada prevalência de sintomatologia osteomuscular em $63,9 \%$ da amostra ${ }^{18}$.

A tabela 4 apresenta que $48,9 \%$ das pessoas associam três problemas osteomusculares ao trabalho e que $20 \%$ associam quatro problemas. Também a tabela mostra a classificação de DORTs da amostra. Verifica-se que quase a totalidade da amostra ou tem estágio inicial de LER/DORTs $(51,1 \%)$ ou moderado $(44,4 \%)$. 
Tabela 4 - Acometimentos osteomusculares associados ao trabalho e a classificação de LER/DORTs. Caicó, RN, Brasil, 2015

\begin{tabular}{c|c|c|c}
\hline \multicolumn{2}{c|}{ Acometimentos osteomusculares associados ao trabalho } & F & \% \\
\hline \multirow{3}{*}{$\begin{array}{c}\text { Quantidade sintomas que consideram } \\
\text { estar relacionados ao trabalho }\end{array}$} & 1,00 & 1 & 2,2 \\
\cline { 2 - 4 } & 2,00 & 13 & 28,9 \\
\cline { 2 - 4 } & 3,00 & 22 & 48,9 \\
\hline Classificação de LER/DORTs na amostra & 9 & 20,0 \\
\hline Fatores de riscos para LER/ DORTs presentes & F & $\%$ \\
\hline \multicolumn{2}{c|}{ LER/DORTs presente estágio inicial } & 0 & 0,0 \\
\hline \multicolumn{2}{c|}{ LER/DORTs presente estágio moderado } & 23 & 51,1 \\
\hline
\end{tabular}

Fonte: Dados de Pesquisa, 2015.

De maneira semelhante Brandão, Horta e Tomasi ${ }^{19}$ mostraram a partir dos resultados de um estudo realizado com bancários, que $39 \%$ dos entrevistados referiam à ocorrência de dor em três ou mais regiões anatômicas.

Ao avaliar os distúrbios osteomusculares em coletores de lixo, pesquisa evidenciou que $88,2 \%$ da amostra referiam queixas ostiomioarticulares relacionadas ao trabalho ${ }^{20}$. Ressalta-se que a maior desta amostra $(94,5 \%)$ negaram a existência de tais queixas antes de trabalharem como agentes da limpeza urbana ${ }^{20}$.

Quanto aos valores expressos na tabela assemelham-se aos obtidos por Formigoni, Valente e Barboza ${ }^{7}$ que ao avaliar um grupo de comerciantes identificaram a presença de DORT em estágio inicial em 52, 38\% e DORT em estágio moderado em 22,22\% da amostra.

Outra pesquisa ${ }^{18}$ evidenciou que $55 \%$ dos operadores de máquinas de colheita florestal analisados apresentaram sintomatologia leve, enquanto o restante de moderado ao forte.

Outro dado proveniente desta pesquisa com lavadeiras referiu-se à correlação entre LER/DORTs, a idade, a renda e o tempo de trabalho. Apenas a idade se correlacionou significativamente e positivamente com a LER/DORTs $(\rho=0,30$; $p \leq 0,05)$, mostrando que quanto maior a idade maior a pontuação na medida de DORT/LER.

A correlação direta entre a idade elevada e a presença de DORT/LER foi também observada em estudo com um grupo de professores ${ }^{21} \mathrm{e}$ mineiros ${ }^{22}$.

O processo biológico de envelhecimento por si só, é responsável pelo comprometimento morfofuncional do sistema musculoesquelético, por conseguinte, torna mais abrangente a probabilidade de ocorrência ou agravamento dos distúrbios osteomusculares relacionados ao trabalho ${ }^{23}$. As DORTs configuram-se como consequências tardias, decorrentes do uso errôneo e repetitivo dos membros superiores e das regiões a eles adjacentes, estando, assim, passíveis ao agravamento conforme o passar dos anos ${ }^{24}$.

Por fim, como limitações desta pesquisa ressalta-se a carência de estudos com lavadeiras, o que impossibilitou melhor discussão dos achados. Adicionalmente, o tamanho da amostra pode ter sido um fator limitante, embora tenha participado da pesquisa $90 \%$ das trabalhadoras do município de Caicó-RN e cadastradas na Prefeitura Municipal local.

\section{Considerações Finais}

Observou-se a exclusividade de mulheres trabalhadoras e constatou-se a maior frequência de acometimento por distúrbios osteomusculares nas regiões de mãos/punho e cotovelos, respectivamente. Também, todas as lavadeiras apresentaram ao menos uma queixa. Quanto aos problemas osteomusculares relacionados ao trabalho, o mais associado foi o dos punhos ou mãos, seguido dos cotovelos e da região do pescoço/região cervical. 
Embora tenha sido evidenciada a presença de queixas osteomusculares relacionadas ao trabalho na totalidade da amostra, observando-se a existência de mais de uma queixa, não foi constatada relação entre nível de escolaridade ou renda e a presença dos distúrbios. Ademais, durante a coleta de dados foi identificada a dificuldade que as profissionais encontram para buscar tratamentos de saúde, uma vez que estes envolveriam o afastamento das atividades laborais, ao menos temporário, culminando, por conseguinte, no comprometimento da renda mensal.

Assim, é imprescindível que os órgãos municipais de saúde, especialmente o Centro de Referência em Saúde do Trabalhador, desenvolvam campanhas preventivas e de promoção da saúde com enfoque na melhoria das condições de saúde gerais das lavadeiras, com recomendações gerais e sobre a necessidade de serem observadas pausas durante a jornada de trabalho. Também, os fisioterapeutas atuantes no Núcleo de Apoio à Saúde da Família (NASF) local podem adotar programa de ginástica laboral.

\section{Referências}

1. Marx K. O capital. Rio de Janeiro: Civilização Brasileira; 1968.

2. Saldanha JHS, Pereira APM, Neves RF, Lima MAG. Facilitadores e barreiras de retorno ao trabalho de trabalhadores acometidos por LER/DORT. Rev. bras. saúde ocup. 2013;38(127):122-38.

3. Felli VEA. Condições de trabalho de enfermagem e adoecimento: motivos para a redução da jornada de trabalho para 30 horas. Enffoco, 2012;3(4):178-81.

4. Siqueira ACA, Couto MT. As LER/DORT no contexto do encontro simbólico entre pacientes e médicos peritos do INSS/ SP. Saude soc. 2013;22(3):714-26.

5. Brasil. Ministério da Saúde. Dor relacionada ao trabalho: lesões por esforços repetitivos (LER): distúrbios osteomusculares relacionados ao trabalho (DORT). Brasília: Editora do Ministério da Saúde; 2012.

6. Cunha WT, Freitas MCS.Nas mãos das charuteiras, histórias de vida e de LER/DORT. Rev. baiana saúde pública. 2011; 35(1):159-74.

7. Formigoni PGP, Valente FM, Barboza MAI. Fatores de risco para distúrbios osteomusculares relacionados ao trabalho em funcionários de centro comercial. RevlnstCiênc Saúde. 2008; 26(2): 207-9.

8. Pinheiro FA, Troccoli BT, Carvalho, C. V. Validação do Questionário Nórdico de Sintomas Osteomusculares como medida de morbidade. Rev. Saúde Públ. 2002; 36(3):307-12.

9. GaleazzilMS, Garcia L, Driemeier MM, Toni M, Kreling NH, Follador P. Mulheres trabalhadoras: 10 anos de mudanças no mercado de trabalho atenuam desigualdades.Mulher e Trabalho. 2003; 3:9-35.

10. Anunciação CGM, Sales LA, Andrade MC, Silveira CA, Paiva SMA. Sinais e Sintomas osteomusculares relacionadas ao trabalho em profissionais de enfermagem. Saúde (Santa Maria). 2016; 42(2):9-17.

11. França DXS, Bakke HA. Queixas Musculoesqueléticas em Mototaxistas. Rev. de Atenção à Saúde. 2015;13(45):26-33.

12. Lima MAG, Andrade AGM, Bulcão CMA, Mota EMCL, Magalhães FB, Carvalho RCP et al. Programa de reabilitação de trabalhadores com LER/DORT do Cesat/Bahia: ativador de mudanças na saúde do trabalhador. Rev. bras. saúde ocup. 2010; 35(121):112-21.

13. Pereira EF, Teixeira CS, Santos A. Qualidade de vida: abordagens, conceitos e avaliação. Rev. Bras. Educ. Fís. Esportes. 2012; 26(2):241-50.

14. Moura AL, Reis LM, Vannuchi MTO, Haddad MCL, Domansky RC. Capacidade para o trabalho de funcionários da prefeitura de um campus universitário público. Rev. Eletr. Enf. 2013;15(1):130-7.

15. Guimarães BM, Azevedo LS. Riscos de distúrbios osteomusculares em punhos de trabalhadores de uma indústria de pescados. Fisioter. mov. 2013; 26(3): 488-9.

16. Isosaki M, Cardoso E, Glina DMR, Alves ACC, Rocha LE. Prevalência de Sintomas osteomusculares entre trabalhadores de um Serviço de Nutrição Hospitalar em São Paulo, SP. Rev. bras. Saúde ocup. 2011; 36(124): $238-46$.

17. Lelis CM, Battaus MRB, Freitas FCT, Rocha FLR, Marziale MHP, Robazzi MLCC. Distúrbios osteomusculares relacionados ao trabalho em profissionais de enfermagem: revisão integrativa da literatura. Acta paul. enferm. 2012; 25(3): 477-82, 2012.

18. Silva EP, Minette LJ, Sanches ALP, Souza AP, Silva FL, Mafra SCT. Prevalência de Sintomas Osteomusculares em operadores de máquinas de colheita florestal. Rev. Árvore. 2014;38(4):739-45. 
19. Brandão AG, Horta BL, Tomasi E. Sintomas de distúrbios osteomusculares em bancários de Pelotas e região: prevalência e fatores associados. Rev. bras. epidemiol.2005; 8(3): 295-305.

20. Cardoso RK, Rombaldi AJ, Silva MC. Distúrbios osteomusculares e fatores associados em coletores de lixo de duas cidades de porte médio do Sul do Brasil. Rev.dor. 2014; 15(1):13-6.

21. Cardoso JP, Ribeiro IQB, Araújo TM, Carvalho FM, Reis EJFB. Prevalência de dor musculoesquelética em professores. Rev. bras. epidemiol.2009; 12(4):604-14.

22. Sousa MNA, Santos BMO, Zaia JE, Bertoncello D, Feitosa ANA, Assis EV et al. Musculoskeletaldisorders in informal mining workers. IAM. 2015;8 (183):1-8.

23. Borges A. Alterações neuromusculares e funcionais decorrentes do envelhecimento e da osteoartrite e suas consequências para mudanças na qualidade de vida em idosos. [monografia]. Porto Alegre: UFRGS; 2011.

24. Ribeiro NF, Fernandes RCP, Solla DJF, Santos Junior AC, Sena Junior AS. Prevalência de distúrbios osteomusculares relacionados ao trabalho em profissionais de enfermagem. Rev. bras. epidemiol. 2012; 15(2):429-38.

\author{
Elenir Alcântara Diniz \\ Endereço para correspondência - Rua: Severino Soares, SN, Q13, L08, \\ Condomínio Vilas do Lago, CEP: 58701-380, Patos, PB, Brasil. \\ E-mail: enfermeira.elenir@outlook.com \\ Lattes: http://lattes.cnpq.br/3843454726227308 \\ Rafaella do Carmo Ribeiro - rafaellaribeiro_@hotmail.com \\ Milena Nunes Alves de Sousa - minualsa@hotmail.com
}

Enviado em 02 de setembro de 2016. Aceito em 18 de julho de 2017. 